\title{
Prospects of testing an UHECR single source class model with the K-EUSO orbital telescope
}

\author{
Oleg Kalashev ${ }^{1}$, Maxim Pshirkov ${ }^{1,2,3}$, and Mikhail Zotov ${ }^{4}$ \\ ${ }^{1}$ Institute for Nuclear Research of the Russian Academy of Sciences, Moscow, 117312, Russia \\ ${ }^{2}$ Sternberg Astronomical Institute, Lomonosov Moscow State University, Moscow, 119992, Russia \\ ${ }^{3}$ Lebedev Physical Institute, Pushchino Radio Astronomy Observatory, 142290, Russia \\ ${ }^{4}$ Skobeltsyn Institute of Nuclear Physics, Lomonosov Moscow State University, Moscow, 119991, Russia
}

\begin{abstract}
KLYPVE-EUSO (K-EUSO) is a planned orbital detector of ultra-high energy cosmic rays (UHECRs), which is to be deployed on board the International Space Station. K-EUSO is expected to have a uniform exposure over the celestial sphere and register from 120 to 500 UHECRs at energies above $57 \mathrm{EeV}$ in a 2-year mission. We employed the TransportCR and CRPropa 3 packages to estimate prospects of testing a minimal single source class model for extragalactic cosmic rays and neutrinos by Kachelrieß, Kalashev, Ostapchenko and Semikoz (2017) with K-EUSO in terms of the large-scale anisotropy. Nearby active galactic nuclei Centaurus A, M82, NGC 253, M87 and Fornax A were considered as possible sources of UHECRs. We demonstrate that an observation of more than 200 events will allow testing predictions of the model with a high confidence level providing the fraction of events arriving from any of the sources is $\simeq 10-15 \%$, with a smaller contribution for larger samples. These numbers agree with theoretical expectations of a possible contribution of a single source in the UHECR flux. Thus, K-EUSO can provide good opportunities for verifying the minimal model basing on an analysis of the large-scale anisotropy of arrival directions of UHECRs.
\end{abstract}

\section{Introduction}

Ultra-high energy cosmic rays (UHECRs) with energies above $\sim 50 \mathrm{EeV}$, sometimes called extreme-energy cosmic rays, were first registered almost 60 years ago [1] but their nature and sources still remain an open problem of astrophysics and cosmic ray physics. UHECRs are supposed to be produced in extragalactic sources, and this has been recently corroborated by an observational finding of the dipole anisotropy in the arrival directions of UHECRs with energies above $8 \mathrm{EeV}$ [2]. Different classes of astrophysical objects are considered as possible sources of UHECRs, among them gamma-ray bursts [3-5], young millisecond pulsars and magnetars [6-8], tidal disruption events [911], active galactic nuclei (AGN) of different types [12] and mechanisms of acceleration, including blazars [13], black hole jets [14-16] and a few other, see, e.g., [17] for a more in-depth discussion.

Recently, a minimal single source class model for extragalactic cosmic rays and neutrinos was proposed by Kachelrieß, Kalashev, Ostapchenko and Semikoz [18] (KKOS in what follows), which can explain the observed energy spectrum and mass composition of cosmic rays (CRs) with energies above $\sim 10^{17} \mathrm{eV}$, but also matches the high-energy neutrino flux measured by IceCube. The scenario assumes that UHECRs are produced by (possibly a subclass of) AGN. The model neglects the acceleration process details and just relies on the following basic assumptions: i) the energy spectra of nuclei after the accel- eration phase follow a power-law with a rigidity dependent cutoff

$$
j_{\mathrm{inj}}(E) \propto E^{-\alpha} \exp \left[-E /\left(Z E_{\max }\right)\right] ;
$$

ii) the $\mathrm{CR}$ nuclei diffuse first through a zone dominated by photo-hadronic interactions, and then they escape into a second zone dominated by hadronic interactions with gas. It was shown that a good fit to the CR energy spectrum can be obtained assuming only hadronic interactions of UHECRs with gas around their sources, but it is difficult to reproduce the observed distribution of $X_{\max }$ of CR-induced air showers. It is possible to reduce significantly the fraction of heavy nuclei in the primary fluxes and therefore fit satisfactorily both the spectrum and composition data on UHECRs only after adding photo-nuclear interactions with a relatively large interaction depth, which suggests that UHECRs are accelerated close to a supermassive black hole. Moreover, the secondary high-energy neutrino flux obtained in the scenario matches the IceCube measurements [19], while the contribution of unresolved UHECR sources to the extragalactic $\gamma$-ray background [20] is of the order of $30 \%$.

The spectrum of CR leaving the source environment (i.e., after passing the second zone) in the best fit obtained by the KKOS model with $\alpha=1.5$ is shown in the top panel of Fig. 1 for several mass ranges. The attenuation effect on the integral flux is illustrated in the bottom panel by plotting the distance at which the total flux above the given energy drops by factor of $1 / e$. Note that the integral 
flux attenuation length depends both on the initial source spectrum and composition.

Due to strong propagation effects, the observable cosmic ray mass composition and energy spectrum can vary considerably for each individual source even though the injection spectrum is precisely the same for all of them. Moreover, at energies $E \simeq 150 \mathrm{EeV}$, where attenuation length for the integral flux drops to tens of Mpc, the CR flux may be dominated by the contribution of a nearby source located within $20 \mathrm{Mpc}$ from the Milky Way. This, in turn, can lead to a noticeable anisotropy in arrival directions of UHECRs of sufficiently high energies. Orbital detectors with a sufficiently large exposure will provide good opportunities for studying this effect due to their possibly almost uniform exposure of the whole celestial sphere [21-23].
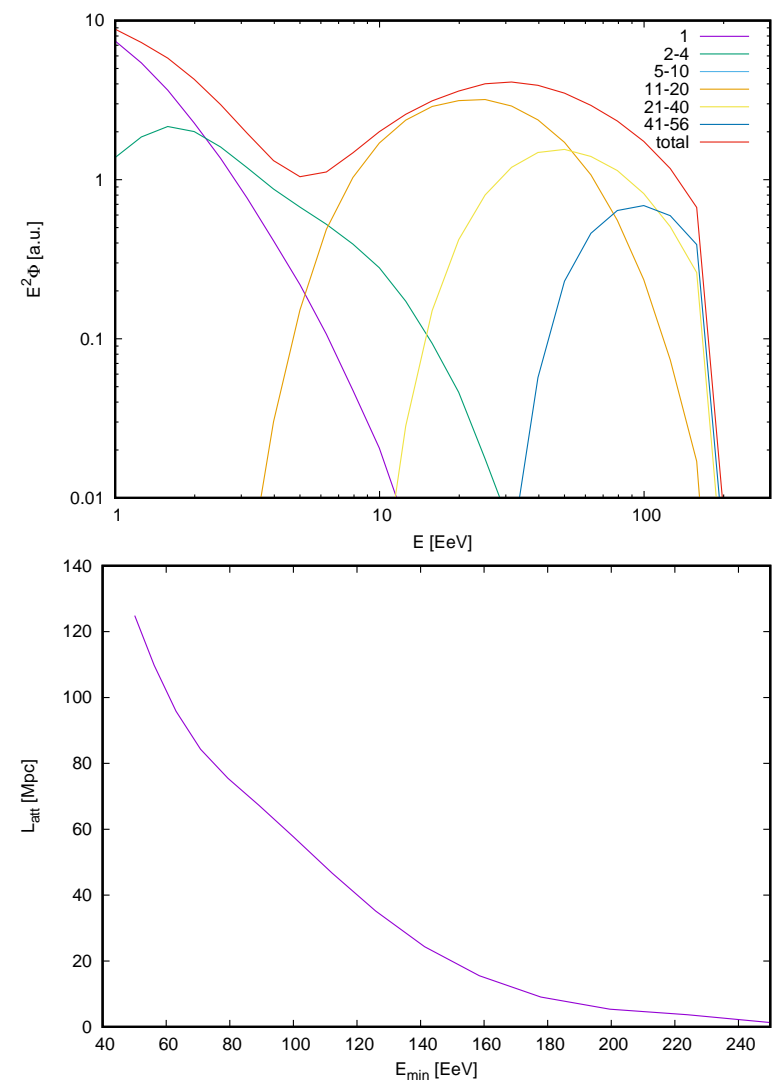

Figure 1. The effective CR source energy spectrum for different mass components (top) and integral flux above $E_{\min }$ suppression length (bottom) in the KKOS model.

One of the future orbital experiments that are being actively developed is the KLYPVE-EUSO (K-EUSO) telescope, which is aimed to be installed on board the International Space Station in 2022 for a 2-year mission [2427]. K-EUSO is a further development of a technique of registering UHECRs via ultra-violet radiation emitted by extensive air showers in the atmosphere of Earth from a low-orbit satellite, implemented for the first time in the TUS detector $[28,29]$. The telescope is expected to have a Schmidt-type optical system with the main mirrorreflector of a $4 \mathrm{~m}$ diameter, an entrance pupil of a $2.5 \mathrm{~m}$ diameter and a $1.7 \mathrm{~m}$ focal length. A round-shaped field of view of $40^{\circ}$ will provide an instantaneous geometrical area of nearly $6.7 \times 10^{4} \mathrm{~km}^{2}$ at sea level for an orbit height around $400 \mathrm{~km}$. It is expected that K-EUSO will register from 120 up to almost 500 UHECRs with energies above $57 \mathrm{EeV}$ in two years. The difference between the lower and the upper boundaries of the estimate arises from the difference in the energy spectra of the Pierre Auger Observatory and the Telescope Array [27].

Capabilities of a previous version of the telescope (KLYPVE) to detect the Telescope Array hotspot were studied earlier [30]. In what follows, we study if K-EUSO will be able to verify the KKOS model basing on an analysis of the large-scale anisotropy of arrival directions of UHECRs with energies above $57 \mathrm{EeV}$.

\section{Method of the analysis}

We considered five possible sources of UHECRs that are often discussed in literature and satisfy the KKOS model, namely NGC 253, Centaurus A, M82, M87 and Fornax A. These are radio-loud galaxies located at distances $d \approx$ 3.5 .. $20 \mathrm{Mpc}$ from the Milky Way. The first three sources (NGC 253, Cen A and M82) are the closest among them. They are located at distances $d \approx 3.5 \mathrm{Mpc}$ [31], at different directions in the celestial sphere. M87 (Virgo A) and Fornax A are comparatively distant sources located at $d \approx 18.5 \mathrm{Mpc}$ and $d \approx 20 \mathrm{Mpc}$ respectively. Cen A and Fornax $\mathrm{A}$ are the most luminous among the five sources in the radio band. For each source located at a given distance $d$, we calculated an energy spectrum and mass composition of the CR flux crossing the Milky Way boundary using a public numerical code TransportCR [32], which was also used in the original work [18]. A contribution of other sources was approximated by an isotropic component. The spectrum and mass composition for the isotropic component was calculated by solving the transport equation with a homogeneous source distribution for distances $R>2^{1 / 3} d$, and zero density for smaller distances.

We assumed deflections of CR nuclei in the intergalactic space are small, so that nuclei accelerated at a particular source arrive to the Milky Way within $1^{\circ}$ from the actual direction to the source. The CRPropa 3 package [33] (the GitHub snapshot of 24th June, 2018) was employed to calculate deflections of nuclei in the Galaxy on their way to the Solar system using the Jansson-Farrar model of the Galactic magnetic field (GMF) [34]. All three components of the GMF model (the regular, striated and turbulent ones) were involved in simulations. Backtracking was performed for all possible pairs $(Z, E)$ in the spectra to obtain maps of apparent arrival directions of different nuclei to Earth given original directions of arriving to the Milky Way. Calculations were made on the HEALPix ${ }^{1}$ grid with $N_{\text {side }}=512$ providing an angular resolution of the order of $7^{\prime}$. This is far beyond the angular resolution of the K-EUSO experiment but was chosen to recover accurately an observed distribution of arrival directions of nuclei after their propagation in the Galactic magnetic field.

\footnotetext{
${ }^{1}$ https://healpix.sourceforge.io
} 
There are a number of mathematical tools traditionally used for studying the large-scale anisotropy of cosmic rays. Historically the first one is a harmonic analysis in right ascension, see [2] for a recent application. The effectiveness of the method is mostly confined to the lowest multipoles due to the small statistics of UHECRs. Another approach is based on calculating the angular power spectrum coefficients decomposing the observed flux $f(\theta, \phi)$ into spherical harmonics

$$
\begin{aligned}
a_{\ell m} & =\int f(\theta, \phi) Y_{l}^{m}(\theta, \phi)^{*}, \\
C_{\ell} & =\frac{1}{2 \ell+1} \sum_{m=-\ell}^{+\ell}\left|a_{\ell m}\right|^{2},
\end{aligned}
$$

where $Y_{l}^{m}(\theta, \phi)$ are the spherical harmonics and $a_{\ell m}$ are their multipolar moments (coefficients), see, e.g., [35] for details. Coefficients $C_{\ell}$ provide an efficient tool for estimating deviations from isotropy at different multipoles providing a sufficiently large sample is available.

The IceCube and Auger experiments suggested a simple approach that allows estimating a total impact of different multipoles in deviating from an isotropic distribution of arrival directions and also penalises statistically the search over many angular scales $[36,37]$. They suggested to calculate

$$
D^{2}=\frac{1}{\ell_{\max }} \sum_{\ell=1}^{\ell_{\max }}\left(\frac{C_{\ell, \mathrm{data}}-\left\langle C_{\ell, \text { iso }}\right\rangle}{\sigma_{\ell, \text { iso }}}\right)^{2},
$$

where $C_{\ell \text {,data }},\left\langle C_{\ell \text {,iso }}\right\rangle$ and $\sigma_{\ell \text {,iso }}$ are, respectively, the $C_{\ell}$ observed in the data, and the average and standard deviation from isotropic expectations, all of them calculated at a given scale $\ell$. In practice, $\left\langle C_{\ell \text {,iso }}\right\rangle$ and $\sigma_{\ell \text {,iso }}$ are evaluated using a simulated isotropic flux with the same number of events and exposure as for the data [37]. One can choose a certain confidence level for defining a threshold to accept or reject the isotropy hypothesis and then compare a value of $D^{2}$ calculated for the data to that of $D^{2}$ obtained for the isotropic distribution.

We tried this approach but found another estimator to be slightly more sensitive to deviations from an isotropic distribution. Results presented below are based on

$$
D=\frac{1}{\ell_{\max }} \sum_{\ell=1}^{\ell_{\max }} \frac{C_{\ell, \operatorname{mix}}-\left\langle C_{\ell, \text { iso }}\right\rangle}{\sigma_{\ell, \text { iso }}},
$$

which is the same one as used by the Pierre Auger collaboration but without a square of the summands. This allows one to take into account the fact that the expected deviations from the isotropic case are one-sided (positive). Here, $C_{\ell \text {,data }}$ is replaced with $C_{\ell \text {,mix }}$, which is the $C_{\ell}$ obtained for a simulated mixture of an isotropic flux and UHECRs arriving from a particular source. Contrary to the case when one works with experimental data, we needed to simulate many mixed samples. Then we compared $D$ of the isotropic distribution to the median of the estimator obtained for a mixed flux. Our aim was to find a fraction of events arriving from a perticular source in mixed samples of different sizes such that the probability of deviating from isotropy to occur by chance was $\lesssim 10^{-5}$.

\section{Main results}

We performed simulations for $N_{\mathrm{UHECR}}=100,200, \ldots, 500$ to cover the whole possible range of $N_{\text {UHECR }}$ to be detected by K-EUSO. The main results are presented in Table 1 . The numbers give the percentage of events arriving from a particular source in a sample of size $N_{\mathrm{UHECR}}$, such that the fraction of values of $D$ for the isotropic flux greater than the median of $D$ calculated for mixed samples is $\lesssim 10^{-5}$. For example, in the case of Cen A and $N_{\mathrm{UHECR}}=300$, the median of $D$ equals 1.2 , and nine of 500,000 values of $D$ for the isotropic flux are greater than that, as soon as UHECRs arriving from this source constitute $11 \%$ of the whole sample. This means the large-scale anisotropy observed in this case stands out from isotropic expectations with a $p$-value of $1.8 \times 10^{-5}$. See an illustration in Fig. 2 .

Table 1. Percentage of UHECRs arriving from five candidate sources in samples of size $N_{\mathrm{UHECR}}=100, \ldots, 500$ such that the observed large-scale anisotropy estimated with function $D$ stands out of isotropic expectations with $p$-values $\lesssim 10^{-5}$.

\begin{tabular}{|l|c|c|c|c|c|}
\hline$N_{\text {UHECR }}$ & 100 & 200 & 300 & 400 & 500 \\
\hline NGC 253 & 17 & 12 & 9 & 8 & 7 \\
Cen A & 21 & 14 & 11 & 10 & 9 \\
M82 & 24 & 16 & 13 & 11 & 10 \\
M87 & 27 & 19 & 15 & 13 & 12 \\
Fornax A & 19 & 13 & 10 & 9 & 8 \\
\hline
\end{tabular}

All results presented in Table 1 were obtained for 500,000 isotropic samples and 10,000 mixed samples for each $N_{\mathrm{UHECR}}$. The accuracy of the numbers is $\pm 1 \%$. We tried up to 100,000 mixed samples but simulations revealed that the median of $D$ only weakly depends on the number of samples. An important point to mention is the value of $\ell_{\max }$ used for calculating the estimator $D$. It became clear from simulations and can be seen in the figures below that the coefficients $C_{\ell}$ calculated for mixed samples quickly converge to isotropic values, so that it makes no sense to take into account a contribution from multipoles with $\ell>16$. The estimator becomes even more sensitive with lower $\ell_{\max }$ in case only the lowest multipoles deviate from the isotropic distribution. Still, all results in terms of $D$ presented here were obtained with one and the same $\ell_{\max }=16$ for the sake of uniformity.

Arrival directions of nuclei arriving from the five sources demonstrate strikingly different patterns. The behaviour of $C_{\ell}$ is also different for each of the sources, and the more fuzzy is the pattern of arrival directions of events originated in a given source, the greater is their fraction in the whole sample necessary to distinguish a large-scale anisotropy.

It is clear from Table 1 that the threshold value for the contribution of UHECRs from a single source that can be detected is around $10-15 \%$ for $N_{\mathrm{UHECR}}>200$, and it does not considerably depend either on the source position on the celestial sphere or on the distance to it. Thus, this value can be straightforwardly compared with theoretical expectations. We used the simplest model of identical sources uniformly distributed with a number density $n$. Given that 

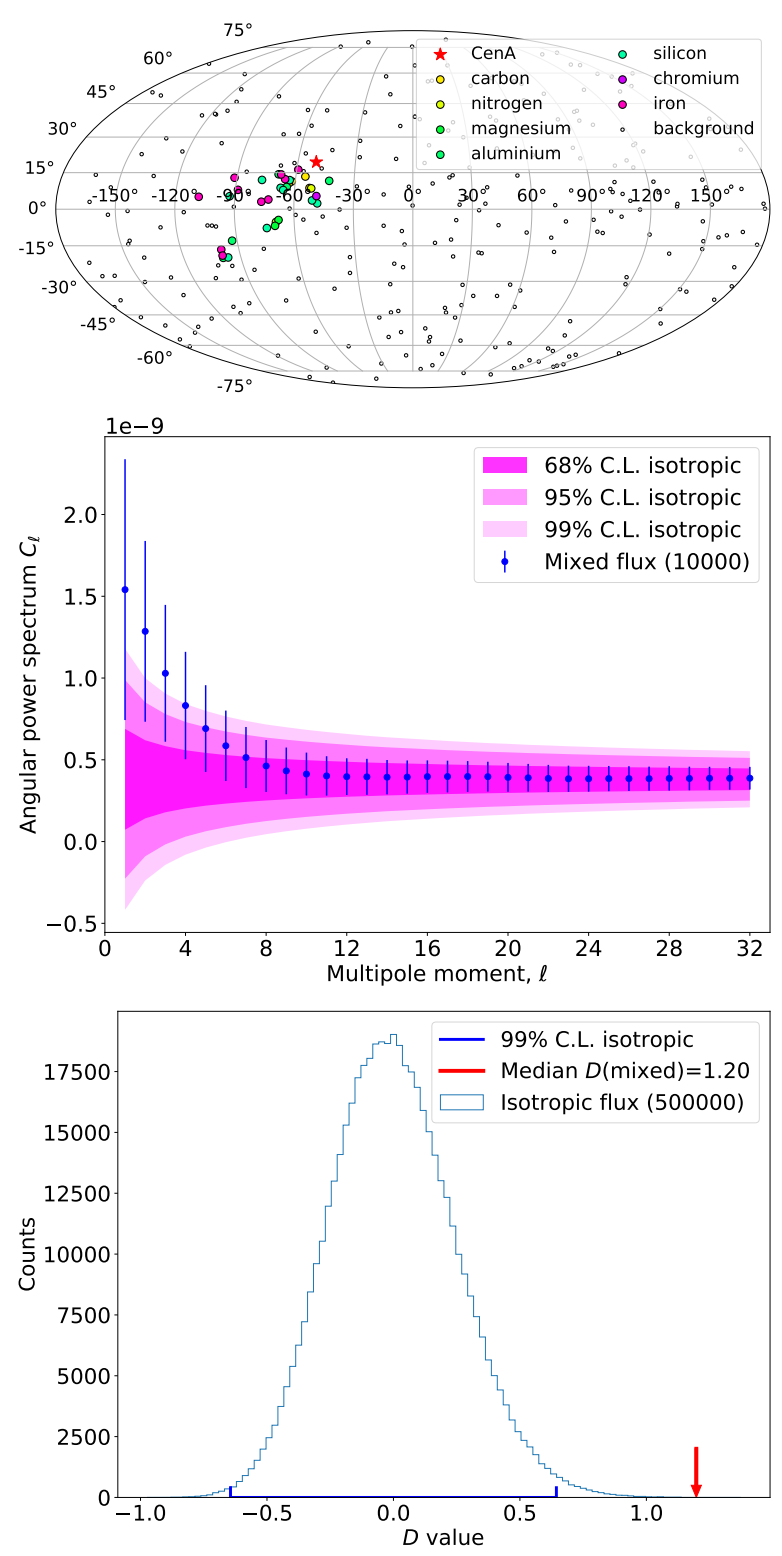

Figure 2. The case of $N_{\mathrm{UHECR}}=300$ and $11 \%$ events coming from Cen A. Top panel: an example of possible arrival directions. UHECRs that form an isotropic background are shown with small open circles. UHECRs arriving from the source are shown with coloured circles according to the type of the respective nucleus. The position of the source is indicated by a star. The map is shown in Galactic coordinates in the Mollweide projection. Middle panel: angular power spectrum $C_{\ell}$ for isotropic and mixed samples. Confidence intervals for $68 \%, 95 \%$ and $99 \%$ levels are shown with different shades of magenta for the isotropic distribution. Blue dots with error bars indicate $C_{\ell}$ for 10,000 mixed samples with $7 \%$ events coming from the source. Bottom panel: the histogram shows the distribution of $D$ for the isotropic flux, with the $99 \%$ confidence interval shown in blue. The red arrow marks the median of $D$ for mixed samples.

the characteristic path length $L_{c}$ at the relevant energies is around $100 \mathrm{Mpc}$ (see Fig. 1), we have performed our simulations in a $V_{\text {box }}=(600 \mathrm{Mpc})^{3}$ box centered at the observer position. Less than $5 \%$ of the total flux comes from outside this box, so we have ignored that part. The total number of simulated sources was equal to $N_{\text {src }}=n V_{\text {box }}$.
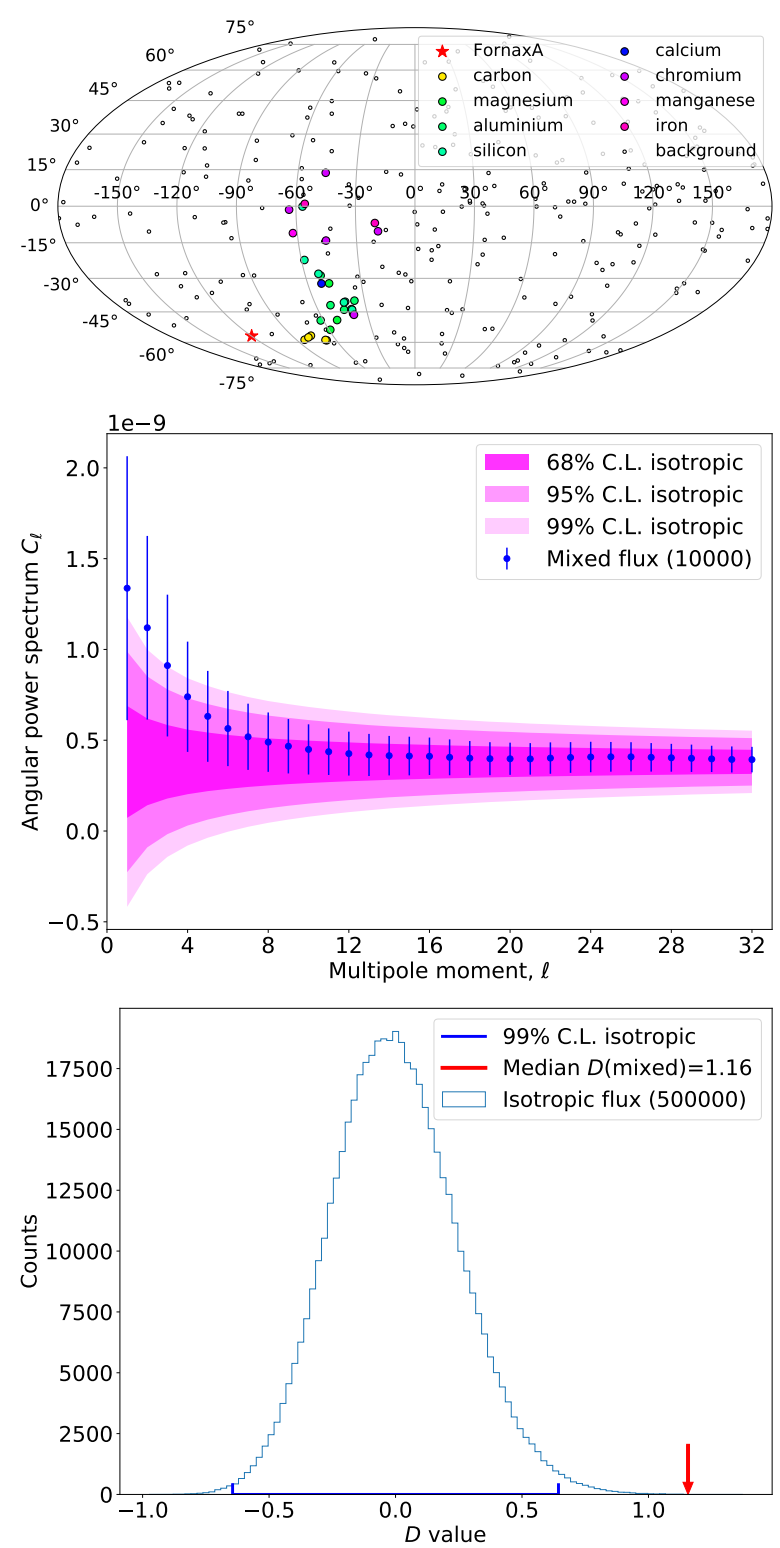

Figure 3. The case of $N_{\mathrm{UHECR}}=300$ and $10 \%$ events coming from Fornax A. See the caption of Fig. 2 for other details.

The contribution from an individual source located at distance $d$ was calculated as

$$
\Phi=\exp \left(-d / L_{c}\right) / d^{2}
$$

Finally, all contributions were summed up, and fractions from the brightest (the closest in this set up) and the second-brightest sources were calculated. This procedure was repeated 100,000 times and that gave a fairly good sampling of the distributions (see Fig. 4). The median values of these distributions for different values of density $n$ are presented in Table 2. It can be seen that the analysis of a large scale anisotropy observed by K-EUSO will have a potential to constrain the density of identically distributed sources at the level $n>(1-2) \times 10^{-5} \mathrm{Mpc}^{-3}$. This value shall be compared with the current limits coming from the non-observation of significant clustering at intermediate angular scales in the same energy range by the Pierre Auger Observatory: $n>6 \times 10^{-6} \mathrm{Mpc}^{-3}$ [38]. 


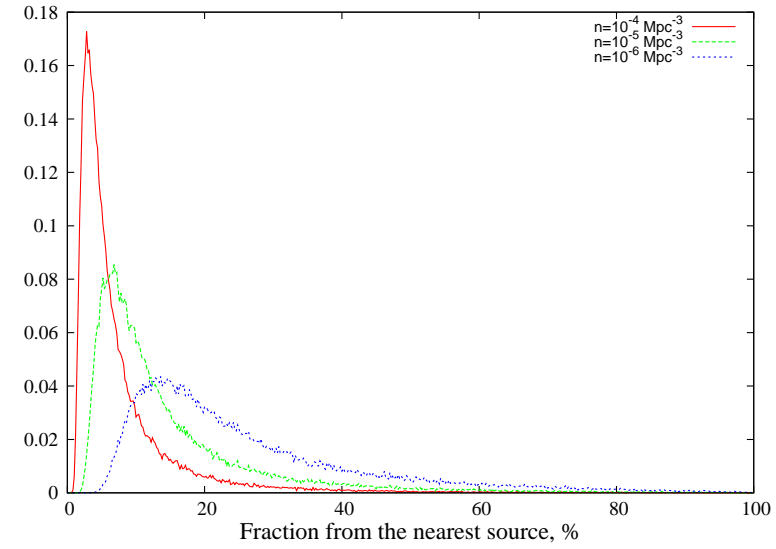

Figure 4. Distribution of percentage of the closest source contribution for different values of the source density.

Table 2. Percentage of UHECRs with $E>57 \mathrm{EeV}$ arriving from the closest and the second closest source in the setup of identical uniformly distributed sources with density $n$.

\begin{tabular}{|l|c|c|}
\hline$n, \mathrm{Mpc}^{-3}$ & Closest & Second closest \\
\hline $10^{-4}$ & 5.2 & 1.8 \\
$3 \times 10^{-5}$ & 7.5 & 2.7 \\
$10^{-5}$ & 10.6 & 3.9 \\
$3 \times 10^{-6}$ & 15.0 & 5.0 \\
$10^{-6}$ & 20.9 & 6.3 \\
\hline
\end{tabular}

\section{Conclusions}

We have studied if a minimal single source class model for extragalactic CRs and neutrinos suggested by Kachelrieß, Kalashev, Ostapchenko and Semikoz [18] can be verified with the future K-EUSO orbital detector basing on an analysis of a large-scale anisotropy of arrival directions of UHECRs with energies above $57 \mathrm{EeV}$. We considered five possible sources often discussed in literature and allowed by the model (Centaurus A, M82, NGC 253, M87 and Fornax A), focusing on the case of a single source providing a deviation from an otherwise isotropic distribution. Using extensive simulations performed with the TransportCR and CRPropa 3 packages, we found that the answer strongly depends on the number of UHECRs registered by K-EUSO and the fraction of events coming from one of the sources in the whole sample. The main result calculated for the $p$-value $\lesssim 10^{-5}$ and the size of samples from 100 to $500 \mathrm{CRs}$ is presented in Table 1. It demonstrates that the K-EUSO experiment will be able to distinguish a large-scale anisotropy arising in the KKOS model from any of the above sources (assuming the JanssonFarrar model of the Galactic magnetic field [34]) with the given $p$-value providing the detector registers more than 200 events and the fraction of CRs arriving from a particular source is $\simeq 10-15 \%$ with a smaller contribution for larger samples.

This research has made use of the NASA/IPAC Extragalactic Database (NED), which is operated by the Jet Propulsion Laboratory, California Institute of Technology, under contract with the
National Aeronautics and Space Administration, and of the SIMBAD database, operated at CDS, Strasbourg, France [39]. We have employed IPython [40] to perform calculations and Matplotlib [41] to make figures. Some of the results in this paper have been derived using the HEALPix package [42]. The work was done with partial financial support from the Russian Foundation for Basic Research grant No. 16-29-13065.

\section{References}

[1] J. Linsley, L. Scarsi, B. Rossi, Physical Review Letters 6, 485 (1961)

[2] Pierre Auger Collaboration, A. Aab, P. Abreu, M. Aglietta, I.A. Samarai, I.F.M. Albuquerque, I. Allekotte, A. Almela, J. Alvarez Castillo, J. Alvarez-Muñiz et al., Science 357, 1266 (2017), 1709.07321

[3] E. Waxman, Phys. Rev. Lett. 75, 386 (1995)

[4] M. Milgrom, V. Usov, The Astrophysical Journal Letters 449, L37 (1995), astro-ph/9505009

[5] M. Vietri, Astrophysical Journal 453, 883 (1995), astro-ph/9506081

[6] P. Blasi, R.I. Epstein, A.V. Olinto, The Astrophysical Journal Letters 533, L123 (2000), astro-ph/9912240

[7] J. Arons, Astrophysical Journal 589, 871 (2003), astro-ph/0208444

[8] K. Fang, K. Kotera, A.V. Olinto, Astrophysical Journal 750, 118 (2012), 1201. 5197

[9] G.R. Farrar, A. Gruzinov, Astrophysical Journal 693, 329 (2009), 0802 . 1074

[10] G.R. Farrar, in European Physical Journal Web of Conferences (2012), Vol. 39 of European Physical Journal Web of Conferences, p. 07005, 1210. 1232

[11] C. Guépin, K. Kotera, E. Barausse, K. Fang, K. Murase, Astronomy \& Astrophysics 616, A179 (2018), 1711.11274

[12] A. Pe'er, K. Murase, P. Mészáros, Physical Review D 80, 123018 (2009), 0911. 1776

[13] K. Murase, C.D. Dermer, H. Takami, G. Migliori, Astrophysical Journal 749, 63 (2012), 1107. 5576

[14] C.D. Dermer, S. Razzaque, J.D. Finke, A. Atoyan, New Journal of Physics 11, 065016 (2009), Q811.1160

[15] K. Fang, K. Murase, Nature Physics 14, 396 (2018), 1704.00015

[16] S.S. Kimura, K. Murase, B.T. Zhang, Physical Review D 97, 023026 (2018), 1705.05027

[17] K. Kotera, A.V. Olinto, Annu. Rev. Astron. Astrophys. 49, 119 (2011), 1101.4256

[18] M. Kachelrieß, O. Kalashev, S. Ostapchenko, D.V. Semikoz, Phys. Rev. D96, 083006 (2017), 1704.06893

[19] M.G. Aartsen et al. (IceCube), Astrophys. J. 833, 3 (2016), 1607.08006

[20] M. Ackermann et al. (Fermi-LAT), Astrophys. J. 799, 86 (2015), 1410. 3696 
[21] B. Rouillé d'Orfeuil, D. Allard, C. Lachaud, E. Parizot, C. Blaksley, S. Nagataki, Astronomy \& Astrophysics 567, A81 (2014), 1401. 1119

[22] F. Oikonomou, K. Kotera, F.B. Abdalla, Journal of Cosmology and Astroparticle Physics 1, 030 (2015), 1409.1925

[23] P.B. Denton, T.J. Weiler, Journal of High Energy Astrophysics 8, 1 (2015), 1505.03922

[24] G.K. Garipov, M.Y. Zotov, P.A. Klimov, M.I. Panasyuk, O.A. Saprykin, L.G. Tkachev, S.A. Sharakin, B.A. Khrenov, I.V. Yashin, Bull. Rus. Acad. Sci. Physics 79, 326 (2015)

[25] M. Panasyuk, P. Klimov, B. Khrenov, S. Sharakin, M. Zotov, P. Picozza, M. Casolino, T. Ebisuzaki, P. Gorodetzky, in 34th International Cosmic Ray Conference (ICRC2015) (2015), Vol. 34, p. 669, https://pos.sissa.it/236/669/pdf

[26] P. Klimov, M. Casolino, the JEM-EUSO Collaboration, in Proceedings, 35th International Cosmic Ray Conference (ICRC 2017): Bexco, Busan, Korea, July 12-20, 2017 (2017), p. 412, https://pos. sissa. it $/ 301 / 412 / \mathrm{pdf}$

[27] M. Casolino, A. Belov, M. Bertaina, T. Ebisuzaki, the JEM-EUSO Collaboration, in Proceedings, 35th International Cosmic Ray Conference (ICRC 2017): Bexco, Busan, Korea, July 12-20, 2017 (2017), p. 368, https://pos. sissa.it/301/368/pdf

[28] P.A. Klimov, M.I. Panasyuk, B.A. Khrenov, G.K. Garipov, N.N. Kalmykov, V.L. Petrov, S.A. Sharakin, A.V. Shirokov, I.V. Yashin, M.Y. Zotov et al., Space Science Reviews 212, 1687 (2017), 1706.04976

[29] B.A. Khrenov, P.A. Klimov, M.I. Panasyuk, S.A. Sharakin, L.G. Tkachev, M.Y. Zotov, S.V. Biktemerova, A.A. Botvinko, N.P. Chirskaya, V.E. Eremeev et al., Journal of Cosmology and Astroparticle Physics 2017, 006 (2017), 1704.07704

[30] D. Semikoz, P. Tinyakov, M. Zotov, Phys. Rev. D93, 103005 (2016), 1601.06363
[31] S. van Velzen, H. Falcke, P. Schellart, N. Nierstenhöfer, K.H. Kampert, Astronomy \& Astrophysics 544, A18 (2012), 1206.0031

[32] O.E. Kalashev, E. Kido, J. Exp. Theor. Phys. 120, 790 (2015), 1406.0735

[33] R. Alves Batista, A. Dundovic, M. Erdmann, K.H. Kampert, D. Kuempel, G. Müller, G. Sigl, A. van Vliet, D. Walz, T. Winchen, Journal of Cosmology and Astroparticle Physics 1605, 038 (2016), 1603.07142

[34] R. Jansson, G.R. Farrar, Astrophys. J. 757, 14 (2012), 1204.3662

[35] O. Deligny, E. Armengaud, T. Beau, P. Da Silva, J.C. Hamilton, C. Lachaud, A. Letessier-Selvon, B. Revenu, Journal of Cosmology and Astroparticle Physics 10, 008 (2004), astro-ph/0404253

[36] J. Hülss, C. Wiebusch, in Proceedings, 30th International Cosmic Ray Conference (ICRC 2007): Merida, Mexico, July 3-11 2007 (2007), 0711.0353

[37] A. Aab et al. (Pierre Auger), Journal of Cosmology and Astroparticle Physics 1706, 026 (2017), 1611.06812

[38] Pierre Auger Collaboration, Journal of Cosmology and Astroparticle Physics 5, 009 (2013), 1305 . 1576

[39] M. Wenger, F. Ochsenbein, D. Egret, P. Dubois, F. Bonnarel, S. Borde, F. Genova, G. Jasniewicz, S. Laloë, S. Lesteven et al., Astronomy and Astrophysics Supplement 143, 9 (2000), astro-ph/0002110

[40] F. Pérez, B.E. Granger, Computing in Science and Engineering 9, 21 (2007)

[41] J.D. Hunter, Computing In Science \& Engineering 9, 90 (2007)

[42] K.M. Górski, E. Hivon, A.J. Banday, B.D. Wandelt, F.K. Hansen, M. Reinecke, M. Bartelmann, Astrophys. J. 622, 759 (2005), astro-ph/0409513 